\title{
Vanishing Theorems on Complete Kähler Manifolds
}

\author{
By \\ Takeo OHSAWA*
}

\section{§ 0 . Introduction}

Let $\mathbf{X}$ be a complex manifold of dimension $n$ and let $\mathbf{E}$ be a holomorphic vector bundle over $\mathbf{X}$. We shall here try to continue the study on the vanishment of the sheaf cohomology groups $\mathrm{H}^{q}(\mathbf{X}$, $\mathcal{O}(\mathbf{E})$ ) which has been performed by Kodaira [10], [11], GrauertRiemenschneider [5], Andreotti-Vesentini [1], [2], Nakano [14], [15], Kazama [9], and others.

The purpose of the present paper is to study the cohomology groups on complete Kähler manifolds. Although the spirit is the same as in [1] and [14], we restrict ourselves to ' $\mathrm{L}^{2}$-cohomology groups' and aim at finding a proper subspace of $\mathrm{L}^{2}$-forms for which $\bar{\partial}$-equation is solvable. We shall prove the following theorem.

$\mathbf{L}^{2}$-vanishing theorem (cf. Theorem 2.8). Let $\mathbf{X}$ be a complete Kähler manifold of dimension $n$, let $(\mathbf{E}, h)$ be a hermitian bundle over $\mathbf{X}$, and let $\sigma$ be a d-closed semipositive $(1,1)$-form on $\mathbf{X}$. Assume that the curvature form for $h$ is equal to or greater than $\sigma$. Then, for any $\bar{\partial}$-closed $\mathbf{E}$-valued $(n, q)$-form $f$ which is square integrable with respect to $\sigma$ (for the definition see Section 2), we can find an $\mathbf{E}$-valued ( $n, q-1)$-form $g$ which is square integrable with respect to $\sigma$ satisfying $\bar{\partial} g=f$. Here $q \geqq 1$.

This is a generalization of theorem 1.5 in [16]. We apply it here to obtain the following two vanishing theorems.

Received January 16, 1982.

* Research Institute for Mathematical Sciences, Kyoto University, Kyoto 606, Japan. 
Theorem (cf. Theorem 3.1). Let $\mathbf{X}$ be a compact Kähler manifold, let $\mathbf{Y}$ be an analytic space, let $f: \mathbf{X} \rightarrow \mathbf{Y}$ be a holomorphic map, and let $(\mathbf{E}, h)$ be a hermitian bundle over $\mathbf{X}$. Assume that the curvature form for $h$ is equal to or greater than the pull-back of a Kähler metric on $\mathbf{Y}$. Then,

$$
\mathrm{H}^{q}\left(\mathbf{Y}, f_{*} \mathcal{O}\left(\mathbf{K}_{\mathbf{x}} \otimes \mathbf{E}\right)\right)=0, \quad \text { for } q \geqq 1 .
$$

Here $\mathbf{K}_{\mathbf{x}}$ denotes the canonical bundle of $\mathbf{X}$ and $f_{*} \mathcal{O}\left(\mathbf{K}_{\mathbf{x}} \otimes \mathbf{E}\right)$ denotes the direct image sheaf of $\mathcal{O}\left(\mathbf{K}_{\mathbf{x}} \otimes \mathbf{E}\right)$.

Theorem (cf. Theorem 4.5). Let $\mathbf{X}$ be a 1-convex manifold with maximal compact analytic set $\mathbf{A}$, and let $\mathbf{E} \rightarrow \mathbf{X}$ be a holomorphic vector bundle. Assume that the restriction of $\mathbf{E}$ to $\mathbf{A}$ is Nakano-semipositive. Then

$$
\mathrm{H}^{q}\left(\mathbf{X}, \quad \mathcal{O}\left(\mathbf{K}_{\mathbf{x}} \otimes \mathbf{E}\right)\right)=0, \quad \text { for } q \geqq 1 .
$$

Fortunately these theorems have applications. Namely, Theorem 3. 1 provides a simple proof of Fujita's semipositivity theorem [3] for relative canonical sheaves, and Theorem 4.5 establishes the converse statement to Laufer's theorem $\mathbf{P}^{1}$ as an exceptional set [13].

The author is very grateful to Prof. K. Diederich who let him work at Gesamthochschule Wuppertal during the preparation of this paper. Sections 4 and 5 were added in Wuppertal. He also thanks the referee for pointing out several mistakes.

\section{§1. Preliminaries}

Let $\mathbf{X}$ be a complex manifold of dimension $n$ with a hermitian metric $\omega$, and let $\mathbf{E} \rightarrow \mathbf{X}$ be a holomorphic vector bundle with a hermitian metric $h$ along the fibers. We say $(\mathbf{E}, h)$ a hermitian bundle over $\mathbf{X}$. We shall regard $\omega$ as a $(1,1)$-form on $\mathbf{X}$, and $h$ as a $C^{\infty}$ section of $\operatorname{Hom}\left(\mathbf{E}, \overline{\mathbf{E}}^{*}\right)$. We denote by $C_{0}^{p, q}(\mathbf{X}, \mathbf{E})$ the space of $\mathbf{E}$-valued $(p, q)$-forms on $\mathbf{X}$ whose supports are compact. The length of $f \in C_{0}^{p, q}(\mathbf{X}, \mathbf{E})$ with respect to $\omega$ and $h$ is denoted by $|f|$. Let $d v$ be the volume form on $\mathbf{X}$ with respect to $\omega$ and set

$$
\|f\|:=\left\{\int_{\mathbf{X}}|f|^{2} d v\right\}^{1 / 2},
$$


which is the usual $\mathrm{L}^{2}$-norm. The $\mathrm{L}^{2}$-norm $\|f\|$ determines a hermitian inner product in $C_{0}^{p, q}(\mathbf{X}, \mathbf{E})$ which we denote by $(f, g)$. Let $\langle f, g\rangle$ be the pointwise inner product with respect to $\omega$ and $h$. Then,

$$
(f, g)=\int_{\mathbf{x}}\langle f, g\rangle d v .
$$

When we need to be more precise, we write $h$ and $\omega$ explicitly, e.g. $\langle f, g\rangle_{h}$ or $\langle f, g\rangle_{h, \omega}$. Let $\mathrm{L}^{p, q}(\mathbf{X}, \mathbf{E}, \omega, h)$ be the completion of $C_{0}^{p, q}(\mathbf{X}, \mathbf{E})$ with respect to the above norm. Then, by the theorem of Riesz-Fischer, $L^{p, q}(\mathbf{X}, \mathbf{E}, \omega, h)$ is naturally identified with the space of $\mathbf{E}$-valued integrable $(p, q)$-forms.

Proposition 1.1. Let $\omega_{1}$ and $\omega_{2}$ be two hermitian metrics satisfying $\omega_{1} \geqq \omega_{2}$. Then,

$$
\|f\|_{\omega_{1}} \leqq\|f\|_{\omega_{2}}, \text { for } f \in C_{0}^{n, q}(\mathbf{X}, \mathbf{E}) \text {. }
$$

Proof. Let $x \in \mathbf{X}$ be any point, and represent $\omega_{1}$ and $\omega_{2}$ at $x$ as follows:

$$
\left\{\begin{array}{l}
\omega_{1}=\sum_{i=1}^{n} \sigma_{i} \bar{\sigma}_{i} \\
\omega_{2}=\sum_{i=1}^{n} \lambda_{i} \sigma_{i} \bar{\sigma}_{i}, \quad \lambda_{i}>0 .
\end{array}\right.
$$

Let $f_{x}$ denote the value of $f$ at $x$. We set

$$
f_{x}=\sum_{\substack{i_{1}<\ldots<i_{p} \\ j_{1}<\ldots<j_{q}}} f_{x_{1} \ldots i_{p} j_{1} . . j_{q}} \sigma_{i_{1}} \wedge \ldots \wedge \sigma_{i_{p}} \wedge \bar{\sigma}_{j_{1}} \wedge \ldots \wedge \bar{\sigma}_{\bar{j}_{q}} .
$$

Then,

Since

$$
\left\{\begin{array}{l}
\left|f_{x}\right|_{\omega_{1}}^{2}=\sum_{\substack{i_{1}<\ldots<i_{p} \\
j_{1}<\ldots<j_{q}}}\left|f_{x i_{1} \cdot i_{p} j_{1} \ldots j_{q}}\right|^{2} \\
\left|f_{x}\right|_{\omega_{2}}^{2}=\sum_{\substack{i_{1}<\ldots<i_{p} \\
j_{1}<\ldots<j_{q}}} \frac{\left|f_{i_{1}} \ldots i_{p} j_{i_{1}} \ldots j_{q}\right|^{2}}{\lambda_{i_{p}} \lambda_{j_{1}} \ldots \lambda_{j_{q}}}
\end{array} .\right.
$$

$$
d v_{\omega_{1}}=\frac{1}{\lambda_{1} \ldots \lambda_{n}} d v_{\omega_{2}} \text { at } x
$$

we have

$$
\left|f_{x}\right|_{\omega_{1}}^{2} d v_{\omega_{1}}=\sum_{\substack{i_{1}<\ldots<i_{p} \\ j_{1}<\ldots<j_{q}}} \frac{\lambda_{i_{1}} \ldots \lambda_{i_{p}} \lambda_{j_{1}} . \lambda_{j_{q}}}{\lambda_{1} \ldots \lambda_{n}}\left|f_{x i_{1} \ldots i_{p} j_{1} \cdot j_{q}}\right|^{2} d v_{\omega_{2} .}
$$


Thus, if $p=n$, then $\lambda_{i_{1}} \ldots \lambda_{i_{p}}$ and $\lambda_{1} \ldots \lambda_{n}$ cancel each other so that

$$
\left|f_{x}\right|_{\omega_{1}}^{2} d v_{\omega_{1}}=\sum_{\substack{i_{1}<\ldots<i i_{p} \\ j_{1}<\ldots<j_{q}}} \lambda_{j_{1}} \ldots \lambda_{j_{q}}\left|f_{x_{1} \ldots i_{p} j_{1} . . j_{q}}\right| d v_{\omega_{2}} .
$$

Since $\lambda_{i}>1$, we obtain from (7),

$$
\left|f_{x}\right|_{\omega_{1}}^{2} d v_{\omega_{1}} \leqq\left|f_{x}\right|_{\omega_{1}}^{2} d v_{\omega_{2}} .
$$

Therefore,

$$
\|f i\|_{\omega_{1}} \leqq i f \|_{\omega_{2}} \cdot \quad \text { Q.E. D. }
$$

As usual we denote by $\bar{\partial}$ the exterior differentiation with respect to the conjugate of the local coordinates of $\mathbf{X}$ and by $\theta\left(=\theta_{\omega, h}\right)$ the adjoint of $\bar{\partial}$ with respect to the inner product of $\mathrm{L}^{p, q}(\mathbf{X}, \mathbf{E}, \omega, h)$. We denote by $L\left(=L_{\omega}\right)$ the multiplication of $\sqrt{-1} \omega$ from the left and by $\Lambda\left(=\Lambda_{\omega}\right)$ the adjoint to $L$. Let $\Theta_{h}$ be the curvature form for $h$. Recall that $\Theta_{h}=\bar{\partial} h^{-1} \partial h$ and that $\Theta_{h}$ is a $\operatorname{Hom}(\mathbf{E}, \mathbf{E})$-valued (1, 1)-form. Thus the left multiplication by $\Theta_{h}$, which we denote by $e\left(\Theta_{h}\right)$, operates on $L^{p, q}(\mathbf{X}, \mathbf{E}, \omega, h)$. The following facts are basic for our purpose.

Proposition 1.2 (cf. [17]). If $\omega$ is a Kähler metric on $\mathbf{X}$, then

$$
\begin{aligned}
& \|\bar{\partial} f\|^{2}+\|\theta f\|^{2} \geqq\left(\sqrt{-1} e\left(\Theta_{h}\right) A f, f\right), \\
& \quad \text { for any } f \in C_{0}^{n, q}(\mathbf{X}, \mathbf{E}) \text {, where } q \geqq 1 .
\end{aligned}
$$

Proposition 1.3 (cf. Theorem 1.1 in [18]). If $\omega$ is a complete hermitian metric on $\mathbf{X}$, then $C_{0}^{p, q}(\mathbf{X}, \mathbf{E})$ is dense in the space $\left\{f \in \mathrm{L}^{p, q}(\mathbf{X}, \mathbf{E}, \omega, h) ; \bar{\partial} f \in \mathrm{L}^{p, q+1}(\mathbf{X}, \mathbf{E}, \omega, h), \theta f \in \mathrm{L}^{p, q-1}(\mathbf{X}, \mathbf{E}, \omega, h)\right\}$ with respect to the norm $\|f\|+\|\bar{\partial} f\|+\|\theta f\|$.

\section{§2. $L^{2}$-Vanishing Theorem}

Let $\mathbf{X}, \omega, \mathbf{E}$ and $h$ be as in Section 1 .

Definition 2.1. Let $\Theta$ be a $\operatorname{Hom}(\mathbf{E}, \mathbf{E})$-valued $(1,1)$-form on $\mathbf{X}$. $\Theta$ is said to be semipositive (positive) if $\Theta$ satisfies

$$
<\Theta(u), u>_{h}(\xi, \bar{\xi}) \geqq 0 \quad(\text { resp. }>0)
$$


for any $u \in \mathbb{E}$ and $\xi \in \mathrm{TX}$ with $u \neq 0$ and $\xi \neq 0$. Here TX denotes the holomorphic tangent bundle of $\mathbf{X}$.

Given two $\operatorname{Hom}(\mathbb{E}, \mathbb{E})$-valued $(1,1)$-forms $\Theta_{1}$ and $\Theta_{2}$, we denote $\Theta_{1} \geqq \Theta_{2}$ if $\Theta_{1}-\Theta_{2}$ is semipositive. A scalar $(1,1)$-form is identified with a $\operatorname{Hom}(\mathbf{E}, \mathbf{E})$-valued $(1,1)$-form when we compare it with $\operatorname{Hom}(\mathbf{E}, \mathbb{E})$-valued forms.

Proposition 2.2. Let $\Theta$ be a semipositive $\operatorname{Hom}(\mathbb{E}, \mathbb{E})$-valued $C^{\infty}$ (1, 1)-form. Then,

$$
\left\langle\sqrt{-1} e(\Theta) \Lambda f, f>_{h} \geqq 0,\right.
$$

for any $f \in C_{0}^{n, q}(\mathbf{X}, \mathbf{E})$.

Proof. The reader is referred to [17].

Definition 2.3. Given a $C^{\infty}$ semipositive $(1,1)$-form $\sigma$ on $X$, we set

$$
\left\{\begin{array}{l}
\mathrm{L}^{n, q}(\mathbf{X}, \mathbb{E}, \sigma, h) \\
:=\left\{f \in \mathrm{L}^{n, q}(\mathbf{X}, \mathbb{E}, \omega+\sigma, h) ; \lim _{\varepsilon \searrow 0}\|f\|_{\varepsilon \omega+\sigma} \text { exists }\right\} \\
\|f\|_{\sigma}:=\lim _{\varepsilon \searrow 0}\|f\|_{\varepsilon \omega+\sigma}, \quad \text { for } f \in \mathrm{L}^{n, q}(\mathbf{X}, \mathbf{E}, \sigma, h) .
\end{array}\right.
$$

Proposition 2.4. $\quad \mathrm{L}^{n, q}(\mathbf{X}, \mathbb{E}, \sigma, h)$ and $\|f\|_{\sigma}$ do not depend on the choice of the metric $\omega$.

Proof. Let $\omega^{\prime}$ be another hermitian metric on $\mathbf{X}$ and let $K$ be any compact subset of $\mathbf{X}$. Then, for any $\varepsilon>0$, we can find $\delta>0$ so that $\varepsilon \omega^{\prime}+\sigma \geqq \delta \omega+\sigma$ on $K$. Hence, in virtue of Proposition 1. 1, we have

$$
\int_{K}|f|_{\varepsilon \omega^{\prime}+\sigma}^{2} d v_{\varepsilon \omega^{\prime}+\sigma} \leqq \int_{K}|f|_{\delta \omega+\sigma}^{2} d v_{\delta \omega+\sigma}
$$

From (14) we observe that if $\lim _{\varepsilon \backslash 0}|| f \|_{\varepsilon \omega+\sigma}$ exists, then $\|f\|_{\varepsilon \omega^{\prime}+\sigma}$ is bounded by $\lim _{\varepsilon \searrow 0}\|f\|_{\varepsilon \omega+\sigma}$. Therefore, $\lim _{\varepsilon \searrow 0}\|f\|_{\varepsilon \omega^{\prime}+\sigma} \leqq \lim _{\varepsilon \searrow 0}\|f\|_{\varepsilon \omega+\sigma}$, which implies independence of $\mathrm{L}^{n, q}(\mathbf{X}, \mathbf{E}, \sigma, h)$ and $\|f\|_{\sigma}$ from the metric $\omega$.

Q. E. D.

Clearly $\mathrm{L}^{n, q}(\mathbf{X}, \mathbb{E}, \sigma, h)$ is a Hilbert space with norm $\|f\|_{\sigma}$ which 
we write $\|f\|$ when there is no fear of confusion.

\section{Definition 2. 5 .}

$$
\left\{\begin{aligned}
\mathrm{N}^{n, q}(\mathbf{X}, \mathbf{E}, \sigma, h):= & \left\{f \in \mathrm{L}^{n, q}(\mathbf{X}, \mathbf{E}, \sigma, h) ; \bar{\partial} f=0\right\} \\
\mathrm{R}^{n, q}(\mathbf{X}, \mathbf{E}, \sigma, h):= & \left\{f \in \mathrm{L}^{n, q}(\mathbf{X}, \mathbf{E}, \sigma, h) ;\right. \text { there exist } \\
& \left.\quad g \in \mathrm{L}^{n, q-1}(\mathbf{X}, \mathbf{E}, \sigma, h) \text { satisfying } \bar{\partial} g=f\right\} \\
\mathrm{H}^{n, q}(\mathbf{X}, \mathbf{E}, \sigma, h):= & \mathrm{N}^{n, q}(\mathbf{X}, \mathbf{E}, \sigma, h) / \mathrm{R}^{n, q}(\mathbf{X}, \mathbf{E}, \sigma, h) .
\end{aligned}\right.
$$

Definition 2.6. $\mathbf{X}$ is called a complete Kähler manifold if there exists a complete Kähler metric on $\mathbf{X}$.

Proposition 2.7. Let $\omega$ be a complete Kähler metric on $\mathbf{X}$. Then,

$$
\|\bar{\partial} f\|^{2}+\|\theta f\|^{2} \geqq\left(\sqrt{-1} e\left(\Theta_{h}\right) \Lambda f, f\right),
$$

for any $f \in \mathrm{L}^{n, q}(\mathbf{X}, \mathbf{E}, \omega, h)$ such that $\bar{\partial} f \in \mathrm{L}^{n, q+1}(\mathbf{X}, \mathbf{E}, \omega, h)$ and $\theta f \in$ $\mathrm{L}^{n, q-1}(\mathbf{X}, \mathbf{E}, \omega, h)$.

Proof is immediate from Proposition 1.2 and Proposition 1.3.

Theorem 2.8. Let $\mathbf{X}$ be a complete Kähler manifold, let $(\mathbf{E}, h)$ be a hermitian bundle over $\mathbf{X}$, and let $\sigma$ be a $d$-closed semipositive $(1,1)$-form on $\mathbf{X}$. If $\Theta_{h} \geqq \sigma$, then

$$
\mathrm{H}^{n, q}(\mathbf{X}, \mathbf{E}, \sigma, h)=0, \text { for } q \geqq 1 \text {. }
$$

Proof. Let $f \in \mathrm{N}^{n, q}(\mathbf{X}, \mathbf{E}, \sigma, h)$. We have to find $g \in \mathrm{L}^{n, q-1}(\mathbf{X}, \mathbf{E}$, $\sigma, h)$ satisfying $\bar{\partial} g=f$. We first fix a complete Kähler metric $\omega$ on $\mathbf{X}$ and prove that for each $\varepsilon>0$ there exists $g_{\varepsilon} \in \mathrm{L}^{n, q-1}(\mathbf{X}, \mathbf{E}, \sigma+\varepsilon \omega, h)$ such that $\bar{\partial} g_{\varepsilon}=f$ and $\left\|g_{\varepsilon}\right\| \leqq C_{q}\|f\|$, where $C_{q}$ is a constant depending only on $q$. In virtue of Hahn-Banach's theorem, the existence of such $g_{\varepsilon}$ is assured by the following estimate:

$$
\begin{aligned}
& \left|(f, u)_{\varepsilon \omega+\sigma}\right|^{2} \leqq C_{q}^{2}|| f||^{2}\left(\|\bar{\partial} u\|^{2}+\|\theta u\|^{2}\right), \\
& \text { for any } u \in \mathrm{L}^{n, q}(\mathbf{X}, \mathbf{E}, \varepsilon \omega+\sigma, h) \text { belonging } \\
& \text { to the domains of } \bar{\partial} \text { and } \theta \text {. }
\end{aligned}
$$

Let $\varphi \in C_{0}^{n, q}(\mathbf{X}, \mathbf{E})$ and let $\delta$ be a positive number less than $\varepsilon$. By Cauchy-Schwarz' inequality we have

$$
\begin{aligned}
& \left|(\varphi, u)_{\varepsilon \omega+\sigma}\right|^{2} \\
& \quad \leqq\left(e(\varepsilon \omega+\sigma) \Lambda_{\delta \omega+\sigma} \varphi, \varphi\right)_{\varepsilon \omega+\sigma}\left(e(\delta \omega+\sigma) \Lambda_{\varepsilon \omega+\sigma} u, u\right)_{\varepsilon \omega+\sigma} \cdot
\end{aligned}
$$


Let $x \in \mathbf{X}$ be any point. We express $\varphi, \sigma+\varepsilon \omega$ and $\sigma+\delta \omega$ at $x$ as follows:

$$
\left\{\begin{array}{l}
\varphi=\sum_{i_{1}<\ldots<i q} \varphi_{i_{1} \ldots i_{q}} \tau_{1} \wedge \ldots \wedge \tau_{n} \wedge \bar{\tau}_{i_{1}} \wedge \ldots \wedge \bar{\tau}_{i_{q}} \\
\sigma+\varepsilon \omega=\sum_{i=1}^{n} \tau_{i} \bar{\tau}_{i}, \\
\sigma+\delta \omega=\sum_{i=1}^{n} \lambda_{i} \tau_{i} \bar{\tau}_{i}, \quad 0<\lambda_{i}<1 .
\end{array}\right.
$$

Then we have

$$
\left\langle e(\varepsilon \omega+\sigma) \Lambda_{\delta \omega+\sigma} \varphi, \varphi>_{\varepsilon \omega+\sigma} d v_{\varepsilon \omega+\sigma}=\sum_{\substack{i_{1}<\ldots<i \\ 1 \leqq \alpha \leqq q}} \frac{\left|\varphi_{i_{1} \ldots i_{q}}\right|^{2}}{\lambda_{i_{\alpha}}} d v_{\varepsilon \omega+\sigma}\right.
$$

and

$$
\langle\varphi, \varphi\rangle_{\delta \omega+\sigma} d v_{\delta \omega+\sigma}=\sum_{i_{1}<\ldots<i_{q}} \frac{\left|\varphi_{i_{1} \ldots i_{q}}\right|^{2}}{\prod_{\alpha=1}^{q} \lambda_{i_{\alpha}}} d v_{\varepsilon \omega+\sigma} .
$$

Comparing (19) and (20) we have

$$
\left\langle e(\varepsilon \omega+\sigma) \Lambda_{\delta \omega+\sigma} \varphi, \varphi\right\rangle_{\varepsilon \omega+\sigma} d v_{\varepsilon \omega+\sigma} \leqq q\langle\varphi, \varphi\rangle_{\delta \omega+\sigma} d v_{\delta \omega+\sigma} .
$$

Therefore,

$$
\int_{\mathbf{X}}\left\langle e(\varepsilon \omega+\sigma) \Lambda_{\delta \omega+\sigma} f, f>_{\varepsilon \omega+\sigma} d v_{\varepsilon \omega+\sigma} \leqq q\|f\|^{2}\right.
$$

Hence,

$$
\left|(f, u)_{\varepsilon \omega+\sigma}\right|^{2} \leqq q|| f||^{2}\left(e(\delta \omega+\sigma) \Lambda_{\varepsilon \omega+\sigma} u, u\right)_{\varepsilon \omega+\sigma} .
$$

Letting $\delta \rightarrow 0$, we have

$$
\left|(f, u)_{\varepsilon \omega+\sigma}\right|^{2} \leqq q\|f\|^{2}\left(e(\sigma) \Lambda_{\varepsilon \omega+\sigma} u, u\right)_{\varepsilon \omega+\sigma} .
$$

By Proposition 2.2 and the assumption that $\Theta_{h} \geqq \sigma$, we have

$$
\left(\sqrt{-1} e(\sigma) \Lambda_{\varepsilon \omega+\sigma} u, u\right) \leqq\left(\sqrt{-1} e\left(\Theta_{h}\right) \Lambda_{\varepsilon \omega+\sigma} u, u\right) .
$$

Note that $\varepsilon \omega+\sigma$ is a complete Kähler metric on $\mathbf{X}$ so that by Proposition 2.7 we have

$$
\left(\sqrt{-1} e\left(\Theta_{h}\right) \Lambda_{\varepsilon \omega+\sigma} u, u\right) \leqq\|\bar{\partial} u\|^{2}+\|\theta u\|_{\left.\right|^{2}}^{2}
$$

Combining (26) with (24) and (25) we obtain (16).

Thus, there exists $g_{\varepsilon} \in \mathrm{L}^{n, q-1}(\mathbf{X}, \mathrm{E}, \varepsilon \omega+\sigma, h)$ satisfying $\bar{\partial} g_{\varepsilon}=f$ and $\left\|g_{\varepsilon}\right\| \leqq q\|f\|$. Note that $\left\|g_{\varepsilon}\right\|_{\omega+\sigma} \leqq\left\|g_{\varepsilon}\right\|$ for $\varepsilon<1$ so that we can choose 
a subsequence of $\left\{g_{\varepsilon}\right\}_{\varepsilon>0}$ converging weakly in $\mathrm{L}^{n, q-1}(\mathbf{X}, \mathbf{E}, \omega+\sigma, h)$. Let the weak limit be $g$. Then we have $\bar{\partial} g=f$. Moreover,

$$
\lim _{\varepsilon \searrow 0}\|g\|_{\varepsilon \omega+\sigma} \leqq \lim _{\varepsilon \searrow 0}\left\|g_{\varepsilon}\right\| \leqq q\|f\| .
$$

Therefore $g \in \mathrm{L}^{n, q-1}(\mathbf{X}, \mathbf{E}, \sigma, h)$.

Q.E.D.

Let us show several examples of (noncompact) complete Kähler manifolds.

Example 1. $\mathbf{C}^{n}$ is a complete Kähler manifold.

Example 2. Every Stein manifold is a complete Kähler manifold. More generally, a Kähler manifold provided with a $C^{\infty}$ exhaustive plurisubharmonic function is a complete Kähler manifold.

Example 3. Given a complete Kähler manifold X,

i) every closed submanifold is a complete Kähler manifold.

ii) Complements of discrete sets are complete Kählerian.

The author does not know whether complements of closed analytic subsets of complete Kähler manifolds are complete Kählerian or not.

Example 4. Let D be a bounded domain with a smooth pseudoconvex boundary in a Kähler manifold. Then, $\mathbf{D}$ is a complete Kähler manifold.

\section{§3. A Generalization of Kodaira's Vanishing Theorem}

Let $\mathbf{Y}$ be a paracompact analytic space over $\mathbf{C}$. By a hermitian metric on $\mathbf{Y}$, we mean a hermitian metric $\sigma$ defined on the regular points of $\mathbf{Y}$ satisfying the following property: for any point $y \in \mathbf{Y}$, there exist a neighbourhood $U$, a holomorphic embedding $\iota: U \rightarrow \mathbb{C}^{N}$ for some $N$, and a $C^{\infty}$ positive $(1,1)$-form $\tilde{\sigma}$ defined on a neighbourhood of $\iota(U)$ for which $\sigma=\imath^{*} \tilde{\sigma}$ on the regular points of $U$. We say $\sigma$ is a Kähler metric if we can choose $\tilde{\sigma}$ to be $d$-closed. For any holomorphic map $f: \mathbf{X} \rightarrow \mathbf{Y}$ from a complex manifold $\mathbf{X}, f^{*} \sigma$ is extended uniquely to a $C^{\infty}$ semipositive $(1,1)$-form on $\mathbf{X}$. We shall not distinguish $f^{*} \sigma$ from its extension. 
Theorem 3.1. Let $\mathbf{X}$ be a compact Kähler manifold, let $f: \mathbf{X} \rightarrow \mathbb{Y}$ be a holomorphic map to an analytic space $\mathbf{Y}$ with a Kähler metric $\sigma$, and let $(\mathbf{E}, h)$ be a hermitian bundle over $\mathbf{X}$. Assume that $\Theta_{h} \geqq f^{*} \sigma$, then

$$
\mathrm{H}^{q}\left(\mathbf{Y}, f_{*} \mathcal{O}\left(\mathbf{K}_{\mathbf{x}} \otimes \mathbf{E}\right)\right)=0, \text { for } q \geqq 1 \text {. }
$$

Before going into the proof we note the following

Lemma 3.2. Let $\pi: \mathbf{X} \rightarrow \mathbf{Y}$ be a holomorphic map between complex manifolds $\mathbf{X}$ and $\mathbf{Y}$ provided with hermitian metrics $\omega_{\mathbf{X}}$ and $\omega_{\mathbf{Y}}$, respectively. Then, for any form $g$ on $\mathbf{Y}$,

$$
\left|\left(\pi^{*} g\right)_{x}\right|_{\omega_{\mathbf{X}}+\pi^{*} \omega_{\mathbf{Y}}} \leqq\left|g_{\pi(x)}\right|_{\omega_{\mathbf{Y}}}
$$

at any point $x \in \mathbf{X}$.

Proof is trivial.

Proof of Theorem 3.1. Let $\mathscr{V}=\left\{V_{i}\right\}_{i \in I}$ be a finite system of Stein open subsets covering of $\mathbf{Y}$ and let $\left\{c_{i_{0}} \ldots i_{q}\right\}$ be a $q$-cocycle of $f^{*} \mathcal{O}\left(\mathbf{K}_{\mathbf{x}} \otimes \mathbf{E}\right)$ associated to $\mathscr{V}(q \geqq 1)$. We set

$$
c_{i_{0} \ldots i_{q}}^{*}=f^{*} c_{i_{0} \ldots i_{q}}{ }^{\circ}
$$

Then $\left\{c_{i_{0} \ldots i_{q}}^{*}\right\}$ is a $q$-cocycle of $\mathcal{O}\left(\mathbb{K}_{\mathbf{X}} \otimes \mathbf{E}\right)$ associated to the covering $\left\{f^{-1}\left(V_{i}\right)\right\}_{i \in I}$. We regard $c_{i_{0} . . i_{q}}^{*}$ as holomorphic $n$-forms on $f^{-1}\left(V_{i_{0}} \cap \ldots\right.$ $\cap V_{i_{q}}$ ) with values in $\mathbf{E}$. Let $\left\{p_{i}\right\}$ be a partition of unity associated to $\mathscr{V}$. We define $\mathbf{E}$-valued $(n, q-k)$-forms $b_{i_{0} . . i_{k-1}}$ on $V_{i_{0}} \cap \ldots \cap V_{i_{k-1}}$ in such a way that

$$
\begin{aligned}
& b_{i_{0} \ldots i_{k-1}} \\
& =\sum_{i_{k} \in I} f^{*} p_{i_{k}} \cdot\left(\bar{\partial}\left(\sum_{i_{k+1} \in I} f^{*} p_{i_{k+1}} \cdot\left(\cdots \bar{\partial} \sum_{i_{q} \in I} f^{*} p_{i_{q}} \cdot c_{i_{0} \cdot i_{q}}^{*}\right) \cdot \cdot\right)\right) \cdot
\end{aligned}
$$

Then we have

$$
\sum_{\alpha=0}^{k-1}(-1)^{i} \bar{\partial} b_{i_{0} \ldots \nu_{\alpha} \ldots i_{k-1}}=0,
$$

and in particular we can define an $\mathbf{E}$-valued $\bar{\partial}$-closed $(n, q)$-form $b$ on $\mathbf{X}$ by $b=\bar{\partial} b_{i}$. By Lemma $3.2\left|\bar{\partial} p_{i}\right|_{\sigma}$ are bounded above. Let $\omega$ be a Kähler metric on $\mathbf{X}$. Then, again by Lemma 3.2, for any $\varepsilon>0$, $\left|\bar{\partial}\left(f^{*} p_{i}\right)\right|_{\varepsilon \omega^{\prime} f^{*} \sigma}$ are bounded above by $\left|\bar{\partial} p_{i}\right|_{\sigma^{\prime}}$. Since $c_{i_{0} \ldots i_{q}}^{*}$ are $(n, 0)-$ 
forms with values in $\mathbf{E},\left|c_{i_{0} \ldots i_{q}}^{*}\right|_{\varepsilon \omega+f^{*} \sigma}^{2} d v_{\varepsilon \omega+f^{*} \sigma}$ are independent of $\varepsilon_{\text {. }}$ Therefore,

$$
\left\{\begin{array}{l}
b \in \mathrm{L}^{n, q}\left(\mathbf{X}, \mathbf{E}, f^{*} \sigma, h\right) \\
b_{i_{0} \ldots i_{k}} \in \mathrm{L}^{n, q-k+1}\left(V_{i_{0}} \cap \ldots \cap V_{i_{k}}, \mathbf{E}, f^{*} \sigma, h\right) .
\end{array}\right.
$$

Thus, in virtue of Theorem 2.8, there exists $a \in \mathrm{L}^{n, q-1}\left(\mathbf{X}, \mathbf{E}, f^{*} \sigma, h\right)$ satisfying $\bar{\partial} a=b$. Let $c_{i}^{*}=b_{i}-a$. Then we have

$$
\left\{\begin{array}{l}
c_{i}^{*} \in \mathrm{L}^{n, q-1}\left(f^{-1}\left(V_{i}\right), \mathbf{E}, f^{*} \sigma, h\right), \\
\bar{\partial} c_{i}^{*}=0 \\
\bar{\partial} b_{i j}=c_{i}^{*}-c_{j}^{*}
\end{array}\right.
$$

Since $V_{i}$ are Stein open sets, $f^{-1}\left(V_{i}\right)$ are complete Kähler manifolds. Hence we can apply Theorem 2.8 to $f^{-1}\left(V_{i}\right)$ and find $a_{i} \in \mathrm{L}^{n, q-2}\left(f^{-1}\right.$ $\left.\left(V_{i}\right), \mathbf{E}, f^{*} \sigma, h\right)$ such that $c_{i}^{*}=\bar{\partial} a_{i}$. Let $c_{i j}^{*}=b_{i j}-a_{i}-a_{j}$. Then we have

$$
\left\{\begin{array}{l}
c_{i j}^{*} \in \mathrm{L}^{n, q-2}\left(f^{-1}\left(V_{i} \cap V_{j}\right), \mathbf{E}, f^{*} \sigma, h\right), \\
\bar{\partial} c_{i j}^{*}=0 \\
\bar{\partial} b_{i j k}=c_{i j}^{*}+c_{j k}^{*}+c_{k i}^{*} .
\end{array}\right.
$$

We can continue this process until we obtain $\mathbf{E}$-valued holomorphic $n$-forms $c_{i_{0} \ldots i_{q-1}}^{*}$ on $f^{-1}\left(V_{i_{0}} \cap \ldots \cap V_{i_{q-1}}\right)$ satisfying

$$
c_{i_{0}, \ldots, i_{q}}^{*}=\sum_{\alpha=0}^{q}(-1)^{\alpha} c_{i_{0}}^{*} \ldots \check{i}_{\alpha} \ldots i_{q} \cdot
$$

We put

$$
c_{i_{0} \cdots i_{q-1}}^{*}=f^{*} c_{i_{0} \ldots i_{q-1}},
$$

where $c_{i_{0} \ldots i_{q-1}}$ are sections of $f_{*} \mathcal{O}\left(\mathbf{K}_{\mathbf{x}} \otimes \mathbf{E}\right)$ over $V_{i_{0}} \cap \ldots \cap V_{i_{q-1}}$. implies that

$$
c_{i_{0} \ldots i_{q}}=\sum_{\alpha=0}^{q}(-1)^{\alpha} c_{i_{0} \ldots \check{i}_{\alpha} \ldots i_{q}} \quad \text { Q. E. D. }
$$

Corollary 3.3 (cf. Fujita [3]). Let $\pi: \mathbf{X} \rightarrow \mathbf{Y}$ be a surjective holomorphic map with connected fibers from a compact Kähler manifold $\mathbf{X}$ to a nonsingular curve $\mathbf{Y}$. Then, every quotient invertible sheaf of $\pi_{*} \omega_{\mathbf{x} / \mathbf{Y}}$ is of nonnegative degree. Here we put $\omega_{\mathbf{x} / \mathbf{Y}}=\mathcal{O}\left(\mathbf{K}_{\mathbf{x}} \otimes \pi^{*} \mathbf{K}_{\mathbf{Y}}^{*}\right)$.

Proof. Let

$$
0 \longrightarrow \mathscr{S} \longrightarrow \pi_{*} \omega_{\mathbf{x} / \mathrm{Y}} \longrightarrow \mathscr{L} \longrightarrow 0
$$


be an exact sequence of coherent analytic sheaves over $\mathbf{Y}$. Let $\mathscr{B}$ be an invertible sheaf of positive degree over $\mathbf{Y}$, then we have the following exact sequence:

$$
\begin{aligned}
& \mathrm{H}^{1}\left(\mathbf{Y}, \mathcal{O}\left(\mathbf{K}_{\mathbf{Y}}\right) \otimes \pi_{*} \omega_{\mathrm{X} / \mathrm{Y}} \otimes \mathscr{B}\right) \\
& \quad \longrightarrow \mathrm{H}^{1}\left(\mathbf{Y}, \mathcal{O}\left(\mathbf{K}_{\mathbf{Y}}\right) \otimes \mathscr{L} \otimes \mathscr{B}\right) \\
& \longrightarrow \mathrm{H}^{2}\left(\mathbf{Y}, \mathcal{O}\left(\mathbf{K}_{\mathbf{Y}}\right) \otimes \mathscr{S} \otimes \mathscr{B}\right) .
\end{aligned}
$$

Since $\operatorname{dim} \mathbf{Y}=1$, we have $\mathrm{H}^{2}\left(\mathbf{Y}, \mathscr{O}\left(\mathbf{K}_{\mathbf{Y}}\right) \otimes \mathscr{S} \otimes \mathscr{B}\right)=0$. On the other hand, by Theorem 3.3,

$$
\mathrm{H}^{1}\left(\mathbf{Y}, \mathcal{O}\left(\mathbf{K}_{\mathbf{Y}}\right) \otimes \pi_{*} \omega_{\mathbf{X} / \mathbf{Y}} \otimes \mathscr{B}\right)\left(=\mathrm{H}^{1}\left(\mathbf{Y}, \pi_{*} \mathcal{O}\left(\mathbf{K}_{\mathbf{x}} \otimes \pi^{*} \mathscr{B}\right)\right)\right)=0 .
$$

Here we used the assumption that the fibers of $\pi$ are connected. Hence $\mathrm{H}^{1}\left(\mathbf{Y}, \mathscr{O}\left(\mathbf{K}_{\mathbf{Y}}\right) \otimes \mathscr{L} \otimes \mathscr{B}\right)$ also vanishes. Therefore $\mathscr{L}$ cannot be an invertible sheaf of negative degree. Otherwise $\mathrm{H}^{1}\left(\mathbb{Y}, \mathcal{O}\left(\mathbb{K}_{\mathbf{Y}}\right) \otimes\right.$ $\left.\mathscr{L} \otimes \mathscr{L}^{*}\right)=0$, which contradicts that $\mathrm{H}^{1}\left(\mathbf{Y}, \mathcal{O}\left(\mathbf{K}_{\mathbf{Y}}\right)\right) \cong \mathrm{H}^{0}\left(\mathbf{Y}, \mathcal{O}_{\mathbf{Y}}\right) \cong \mathbf{C}$.

Q.E.D.

\section{§4. A Vanishing Theorem on 1-Convex Manifolds}

Let $\mathbf{X}$ be a 1-convex manifold, i. e. $\mathbf{X}$ is connected and there exists a $C^{\infty}$ exhaustive function which is strictly plurisubharmonic outside a compact subset of $\mathbf{X}$. The following fact is first due to Grauert [4]: there is a compact analytic subset $\mathbf{A} \subset \mathbf{X}$ and a proper holomorphic map $\pi$ from $\mathbf{X}$ onto a Stein space $\hat{\mathbf{X}}$ such that $\pi_{\mathbf{X}-\mathbf{A}}$ is biholomorphic. If $\mathbf{A}$ is everywhere of positive dimension, $\mathbf{A}$ is called the maximal compact analytic set. By the fundamental work of Hironaka [6], [7], there is a complex manifold $\widetilde{\mathbf{X}}$ obtained from $\widehat{\mathbb{X}}$ by a succession of blowing up along nonsingular centers, such that the induced bimeromorphic map $\pi^{\prime}: \widetilde{\mathbf{X}} \rightarrow \mathbf{X}$ is holomorphic. $\widetilde{\mathbf{X}}$ can be chosen so that

$$
\pi \circ \pi^{\prime} \text { is biholomorphic on } \widetilde{\mathbf{X}}-\pi^{\prime-1}(\mathbf{A}) \text {. }
$$

(II) $\quad \pi^{\prime-1}(\mathbf{A})$ is a divisor with normal crossings whose irreducible components $\left\{\tilde{\mathbf{A}}_{j}\right\}_{j=1}^{\nu}$ are nonsingular.

(III) There exist $\nu$ tuple of positive integers $\left(p_{1}, \ldots, p_{\nu}\right)$ so that the line bundle $\sum_{j=1}^{\nu} p_{j}\left[\tilde{\mathbf{A}}_{j}\right]^{*}$ is very ample.

Set $\tilde{\mathbf{A}}=\sum_{j=1}^{\nu} p_{j} \widetilde{\mathbf{A}}_{j}$ and denote the support of $\widetilde{\mathbf{A}}$ by $|\widetilde{\mathbf{A}}|$. Since $[\widetilde{\mathbb{A}}]^{*}$ is very ample there is a metric $\tilde{a}$ along the fibers of $[\tilde{\mathbf{A}}]^{*}$ such that 
the curvature form $\Theta_{\tilde{a}}$ gives a Kähler metric on $\mathbf{X}$. On $\mathbf{X}-|\widetilde{\mathbf{A}}|, \tilde{a}$ is given by a positive $C^{\infty}$ function $\phi$ satisfying

$$
\partial \bar{\partial}(-\log \psi)=\Theta_{\tilde{a}}
$$

and that

(41) $\quad \log \phi+\log |s|^{2}$ is $C^{\infty}$ on $\mathbf{X}$, where $s$ is a canonical section of $[\tilde{\mathrm{A}}]$.

Via $\pi^{\prime}$ we shall identify $\phi$ with a function on $\mathbf{X}-\mathbf{A}$. Let $\varphi$ be a $C^{\infty}$ plurisubharmonic exhaustive function on $\mathbf{X}$ which is strictly plurisubharmonic outside $\mathbf{A}$.

Proposition 4.1. $\mathbf{X}-\mathbf{A}$ is a complete Kähler manifold.

Proof. Let $V:=\{x \in \mathbf{X}-\mathbf{A} ; \log \psi(x)>0\}$. Then, $V \cup \mathbf{A}$ is a neighbourhood of $\mathbf{A}$ in $\mathbf{X}$. Let $\rho$ be a $C^{\infty}$ function on $\mathbf{X}$ such that $0 \leqq$ $\rho \leqq 1$ on $\mathbf{X}, \rho=0$ on $\mathbf{X}-V$ and $\rho=1$ on a neighbourhood of $\mathbf{A}$. Then, for sufficiently large $K, \partial \bar{\partial}\left(K \varphi^{2}-\log (1+\rho \log \psi)\right)$ is a complete Kähler metric on $\mathbf{X}-\mathbf{A}$.

Q.E.D.

Definition 4.2. Let $\mathbf{Y}$ be an analytic space which is isomorphic to an analytic subset of a domain $\Omega$ in $\mathbf{C}^{n}$ and let $h$ be a $C^{\infty}$ matrixvalued function on $\mathbf{Y}$ with values in $r \times r$ positive definite hermitian matrices. We say that $h$ has semipositive curvature if there is a $C^{\infty}$ extension $\tilde{h}$ of $h$ to a neighbourhood of $\mathbf{Y}$ in $\Omega$ such that $\Theta_{\bar{h}}:=\bar{\partial}\left(\tilde{h}^{-1}\right.$ $\partial \tilde{h}$ ) is semipositive (cf. Definition 2.1).

Proposition 4.3. Let $\pi: \mathbf{Y}^{\prime} \rightarrow \mathbf{Y}$ be a holomorphic map between analytic spaces and let $h$ be a matrix-valued function on $\mathbf{Y}$ with semipositive curvature. Then, $\pi^{*} h$ has semipositive curvature, too.

Proof is trivial.

Definition 4.4. Let $\mathbf{Y}$ be an analytic space and let $(\mathbf{E}, h)$ be a hermitian bundle over $\mathbf{Y} . \quad(\mathbf{E}, h)$ is said to be Nakano-semipositive if for any local representation $h_{i}$ of $h$ as a $C^{\infty}$ matrix-valued function, $h_{i}$ has semipositive curvature.

Theorem 4.5. Let $\mathbf{X}$ be a 1-convex manifold with maximal com- 
pact analytic subset $\mathbf{A}$ and let $(\mathbf{E}, h)$ be a hermitian bundle over $\mathbf{X}$. Assume that $\left(\left.\mathbf{E}\right|_{\mathbf{A}},\left.h\right|_{\mathbf{A}}\right)$ is Nakano-semipositive. Then,

$$
\mathrm{H}^{q}\left(\mathbf{X}, \mathcal{O}\left(\mathbf{K}_{\mathbf{x}} \otimes \mathbf{E}\right)\right)=0, \quad \text { for } q \geqq 1 \text {. }
$$

Proof. First we shall prove that the hermitian bundle $\left(\left.\mathbf{E}\right|_{\mathbf{x}-\mathbf{A}}\right.$, $\left.h(1+\rho \log \phi) e^{-L \varphi}\right)$ is Nakano-semipositive for sufficiently large $L$. Note that by Proposition $4.3\left(\left.\pi^{\prime} * \mathbf{E}\right|_{|\tilde{\mathbf{A}}|},\left.\pi^{\prime} * h\right|_{|\tilde{\mathbf{A}}|}\right)$ is Nakano-semipositive. Since $|\widetilde{\mathbf{A}}|$ is a divisor with normal crossings, it is clear that

$$
\left\langle\Theta_{\pi^{\prime *} h}(u), u>_{\pi^{\prime *} h}(\xi, \bar{\xi}) \geqq 0,\right.
$$

for any $\xi \in\left(\sum_{j=1}^{\nu} \mathrm{T} \tilde{\mathbf{A}}_{j}\right)_{x}$ and $u \in \mathbb{E}_{x}$ at any point $x \in|\widetilde{\mathbf{A}}|$. Here, $T \tilde{\mathbf{A}}_{j}$ are regarded as subspaces of $\mathrm{T} \tilde{\mathbf{X}}$ and

$$
\begin{gathered}
\left(\sum_{j=1}^{\nu} \mathrm{T} \widetilde{\mathbf{A}}_{j}\right)_{x}:=\left\{v \in \mathrm{T}_{x} \widetilde{\mathbf{X}} ;\right. \\
\text { there exist } v_{j} \in \mathrm{T}_{x} \widetilde{\mathbf{A}}_{j}, 1 \leqq \mathrm{j} \leqq \nu \\
\text { such that } \left.v=\sum v_{j}\right\} .
\end{gathered}
$$

We put $\sum_{j=1}^{\nu} \mathrm{T} \tilde{\mathbf{A}}_{j}:=\bigcup_{x \in|\tilde{\mathbf{A}}|}\left(\sum_{j=1}^{\nu} \mathrm{T} \tilde{\mathbf{A}}_{j}\right)_{x}$.

Let $x \in|\widetilde{\mathrm{A}}|$ be any point, let $\left(z_{1}, \ldots, z_{n}\right)$ be a local coordinate on a neighbourhood $U$ of $x$ such that $z_{1} \cdots \cdots z_{k}=0$ is a local equation of $\mid \widetilde{\mathbf{A}}_{\mid}$, and let $\eta$ denote an element of $\mathrm{T} \widetilde{\mathbf{X}}$. Then, $\sum \mathrm{T} \widetilde{\mathbf{A}}_{j}$ is locally defined by the following two equations:

$$
\left\{\begin{array}{l}
\eta\left(z_{1} \cdots \cdots z_{k}\right)=0 \\
z_{1} \cdots \cdot z_{k}=0
\end{array}\right.
$$

Hence we infer from (42) that

$$
\begin{aligned}
& <\Theta_{\pi^{\prime *} h}(u), u>_{\pi^{\prime *} h}(\eta, \bar{\eta}) \\
& \quad \geqq-K|\eta|^{2}|u|^{2}\left(\frac{\mid \eta\left(z_{1} \cdots \cdots z_{k} \mid\right.}{|\eta|}+\left|z_{1} \cdots \cdots z_{k}\right|\right)
\end{aligned}
$$

on $U$, where $K$ depends on $\Theta_{\tilde{a}}, h$ and the choice of $\left(z_{1}, \ldots, z_{n}\right)$. We compare the right hand terms of (45) with $\Theta_{(1+\rho \log \psi)}$ (cf. Proposition 4.1). Since $\log \phi=\infty$ on $|\tilde{\mathbf{A}}|$, there is a neighbourhood $W$ of $|\tilde{\mathbf{A}}|$ such that

$$
\begin{aligned}
& -\partial \bar{\partial} \log (1+\log \phi) \\
& \geqq \frac{-\partial \bar{\partial} \log \psi}{2 \log \psi}+\frac{\partial \psi \bar{\partial} \psi}{2 \psi^{2}(\log \psi)^{2}}
\end{aligned}
$$

on $W-|\tilde{\mathbf{A}}|$. We can find a $C^{\infty}$ function $\lambda$ on $U$ and negative integers $n_{i}$ such that $\phi=\left|z_{1}^{n_{1}} \cdots \cdots z_{k}^{n_{k}}\right|^{2} \lambda$. Shrinking $W$ if necessary we obtain 


$$
\begin{aligned}
\frac{\partial \psi \bar{\partial} \psi}{\phi^{2}(\log \psi)^{2}}+\frac{-\partial \bar{\partial} \log \psi}{\log \phi} & \frac{1}{2(\log \psi)^{2}}\left(\sum_{i=1}^{k} n_{i} \frac{d z_{i}}{z_{i}}+\frac{\partial \lambda}{\lambda}\right)\left(\sum_{i=1}^{k} n_{i} \frac{d \bar{z}_{i}}{\bar{z}_{i}}+\frac{\bar{\partial} \lambda}{\lambda}\right)+\frac{-\partial \bar{\partial} \log \psi}{\log \phi} \\
\geqq & \frac{1}{2(\log \psi)^{2}}\left(\sum_{i=1}^{k} n_{i} \frac{d z_{i}}{z_{i}}\right)\left(\sum_{i=1}^{k} n_{i} \frac{d \bar{z}_{i}}{\bar{z}_{i}}\right) \\
& \quad+\frac{-\partial \bar{\partial} \log \psi}{2 \log \psi}, \text { on } W \cap U-|\tilde{\mathbf{A}}| .
\end{aligned}
$$

Hence

$$
\begin{aligned}
&<\Theta_{\pi^{\prime *} h(1+\rho \log \psi)}(u), u>_{\pi^{\prime *} h}(\eta, \bar{\eta}) \\
& \geqq-K|\eta|^{2}|u|^{2}\left(\frac{\left|\eta\left(z_{1} \cdots \cdot z_{k}\right)\right|}{|\eta|}+\left|z_{1} \cdots \cdot z_{k}\right|\right) \\
&+\frac{1}{4(\log \psi)^{2}}\left(\sum n_{i} \frac{\eta\left(z_{i}\right)}{z_{i}}\right)\left(\sum n_{i} \frac{\overline{\eta\left(z_{i}\right)}}{\bar{z}_{i}}\right) \mid u_{\left.\right|^{2}} \\
&+\frac{1}{4 \log \psi}|\eta|^{2}|u|^{2} \text {, on } W \cap U-|\widetilde{\mathbb{A}}| .
\end{aligned}
$$

From (48) it is easy to see that

$$
\left\langle\Theta_{\pi^{\prime *} h(1+\rho} \log \psi\right)(u), u>_{\pi^{\prime *} h}(\eta, \bar{\eta}) \geqq 0,
$$

on $W \cap U-|\widetilde{\mathbf{A}}|$, where we possibly shrink $U$ and $W$. Thus, by compactness argument $\left(\left.\mathbb{E}\right|_{\mathbf{x}-\mathbf{A}}, h(1+\rho \log \psi) e^{-L \varphi}\right)$ is Nakano-semipositive for sufficiently large $L$. We set $\Phi=(1+\rho \log \phi) e^{-L \varphi}$. Then, by Theorem 2.8, we have

$$
\mathrm{H}^{n . q}\left(\mathbf{X}-\mathbf{A}, \mathbf{E}, \Theta_{\Phi}, h \Phi^{2}\right)=0, \quad \text { for } q \geqq 1 \text {. }
$$

We are going to deduce from (50) that $\mathrm{H}^{q}\left(\mathbf{X}, \mathcal{O}\left(\mathbf{K}_{\mathbf{x}} \otimes \mathbf{E}\right)\right)=0$ for $q \geqq 1$. Let $f$ be any $C^{\infty} \mathbf{E}$-valued $\bar{\partial}$-closed $(n, q)$-form on $\mathbf{X}$. Since any power of $\log \psi$ is locally square integrable on $\mathbf{X}$, we may assume that $f \in \mathrm{L}^{n, q}\left(\mathbf{X}-\mathbf{A}, \mathbf{E}, \Theta_{\Phi}, h \Phi^{2}\right)$, if necessary replacing $\varphi$ by a more rapidly increasing function. Hence we can find $g \in \mathrm{L}^{n \cdot q-1}(\mathbf{X}-\mathbf{A}, \mathbb{E}$, $\left.\Theta_{\Phi}, h \Phi^{2}\right)$ such that $\bar{\partial} g=f$. If $q=1$ we are done, since $g$ is then locally square integrable on $\mathbf{X}$ and in view of the equality $\bar{\partial} g=f$ on $\mathbf{X}-\mathbf{A}, g$ is extended to a $C^{\infty} n$-form with values in $\mathbf{E}$. Let $q \geqq 2$. Then we choose a locally finite covering $\left\{U_{i}\right\}_{i \in I}$ of $\mathbf{X}$ by Stein open sets and define $\left\{f_{i_{1} \ldots i_{k}}\right\},\left\{g_{i_{1} \ldots i_{k}}\right\}$ and $\left\{u_{i_{1} \ldots i_{k}}\right\}$ inductively as follows. Let $u_{i}$ be a $C^{\infty}$ (E-valued) $(n, q-1)$-form on $U_{i}$ such that $\bar{\partial} u_{i}=f$. We set $f_{i}=g-u_{i}$. Since $\bar{\partial} f_{i}=0$ and $f_{i} \in \mathrm{L}^{n, q-1}\left(U_{i}-\mathbf{A}, \mathbb{E}, \Theta_{\Phi}, h \Phi^{2}\right)$, (where we possibly shrink $U_{i}$ and replace $\varphi$ again), we can find $g_{i} \in \mathrm{L}^{n, q-2}\left(U_{i}-\mathbf{A}, \mathbf{E}, \Theta_{\Phi}, h \Phi^{2}\right)$ such that $\bar{\partial} g_{i}=f_{i}$. Assume that $\left\{f_{i_{1} \ldots i_{k}}\right\}$, 
$\left\{g_{i_{1} \ldots i_{k}}\right\}$ and $\left\{u_{i_{1} \ldots i_{k}}\right\}$ are already determined in such a way that

$$
\left\{\begin{array}{l}
\sum_{\alpha=1}^{k}(-1)^{\alpha} g_{i_{1} \ldots i_{\alpha} \cdots i_{k+1}}+\sum_{\alpha=1}^{k}(-1)^{\alpha} u_{i_{1} . . i_{\alpha} \cdot i_{k+1}}=0, \\
\bar{\partial} f_{i_{1} \ldots i_{k}}=0, \\
u_{i_{1} \ldots i_{k}} \text { are } C^{\infty} \text { on } U_{i_{1}} \cap \ldots \cap U_{i_{k}} \\
f_{i_{1} \ldots i_{k}} \in \mathrm{L}^{n, q-k}\left(U_{i_{1}} \cap \ldots \cap U_{i_{k}}-\mathbf{A}, \mathbf{E}, \Theta_{\Phi}, h \Phi^{2}\right), \\
g_{i_{1} \ldots i_{k}} \in \mathrm{L}^{n, q-k-1}\left(U_{i_{1}} \cap \ldots \cap U_{i_{k}}-\mathbf{A}, \mathbf{E}, \Theta_{\Phi}, h \Phi^{2}\right) .
\end{array}\right.
$$

If $k \leqq q-2$, we set $\left\{f_{i_{1} \ldots i_{k+1}}\right\},\left\{g_{i_{1} \ldots i_{k+1}}\right\}$ and $\left\{u_{i_{1} \ldots i_{k+1}}\right\}$ as follows. First we take $u_{i 1 \ldots i_{k+1}}$ to be $C^{\infty}$ and that

$$
\bar{\partial} u_{i_{1} \ldots i_{k+1}}=\sum_{\alpha=1}^{k+1}(-1)^{\alpha+1} u_{i_{1} \ldots i_{\alpha} \ldots i_{k+1}} .
$$

Then we set

$$
f_{i_{1} \ldots i_{k+1}}=\sum_{\alpha=1}^{k+1}(-1)^{\alpha+1} g_{i_{1} \ldots \check{i}_{\alpha \cdot i_{k+1}}}+u_{i_{1} \ldots i_{k+1}} .
$$

We have $\bar{\partial} f_{i_{1} \cdot i_{k+1}}=0$ and may assume that $f_{i_{1} \ldots i_{k+1}} \in \mathrm{L}^{n . q-k-1}\left(U_{i_{1}} \cap \ldots \cap\right.$ $\left.U_{i_{k+1}}-\mathbf{A}, \mathbf{E}, \Theta_{\Phi}, h \Phi^{2}\right)$. Hence we can find $g_{i_{1} \ldots i_{k+1}} \in \mathrm{L}^{n, q-k-2}\left(U_{i_{1}} \cap \ldots\right.$ $\left.\cap U_{i_{k+1}}-\mathbf{A}, \mathbf{E}, \Theta_{\Phi}, h \Phi^{2}\right)$ such that $\bar{\partial} g_{i_{1} \ldots i_{k+1}}=f_{i_{1} \ldots i_{k+1}}$. By the inductive assumption we have

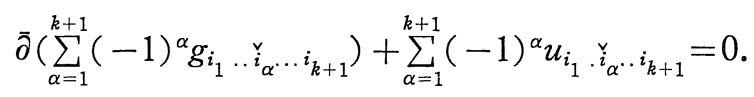

Therefore, for any $k$ with $1 \leqq k \leqq q-1$, we have inductively determined $\left\{f_{i_{1}, i_{k}}\right\},\left\{g_{i_{1}}, i_{k}\right\}$ and $\left\{u_{i_{1}, . i_{k}}\right\}$ satisfying (51). Note that in particular $g_{i_{1}, i_{q-1}}$ are square integrable forms on $U_{i_{1}} \cap \ldots \cap U_{i_{q-1}}$ such that $\bar{\partial}\left(\sum(-1)^{\alpha} g_{i_{1}} . \check{i}_{\alpha} \cdots i_{q}\right)$ are $C^{\infty}$ on $U_{i_{1}} \cap \ldots \cap U_{i_{q}}$. Hence there exist $C^{\infty}$ forms $v_{i_{1} \ldots i_{q-1}}$ on $U_{i_{1}} \ldots U_{i_{q-1}}$ such that

$$
\sum_{\alpha}(-1)^{\alpha} g_{i_{1} \ldots i_{\alpha} \ldots i_{q}}=\sum_{\alpha}(-1)^{\alpha} v_{i_{1} \ldots \nu_{\alpha} \cdots i_{q}} \text {. }
$$

Taking $\bar{\partial}$ of the both sides in (55) we have

$$
\sum_{\alpha}(-1)^{\alpha}\left(u_{i_{1} \ldots \tilde{i}_{\alpha} \ldots i_{q}}+\bar{\partial} v_{i_{1}}, \check{\nu}_{\alpha} \ldots i_{q}\right)=0 .
$$

Therefore, we can find $v_{i_{1} \ldots i_{q-2}}$ such that

$$
u_{i_{1}, i_{q-1}}+\bar{\partial} v_{i_{1} \cdots i_{q-1}}=\sum_{\alpha}(-1)^{\alpha} v_{i_{1} \ldots i_{\alpha} \cdots i_{q-1}},
$$

whence we obtain

$$
\bar{\partial} u_{i_{1} . . i_{q-1}}=\sum_{\alpha}(-1)^{\alpha} \bar{\partial} v_{i_{1}} \ldots \check{i}_{\alpha} \ldots i_{q-1} \cdot
$$

Continuing this process we arrive at the equality 


$$
u_{i}-u_{j}=\bar{\partial} u_{i j}=\bar{\partial} v_{i}-\bar{\partial} v_{j} .
$$

Thus we obtain a $C^{\infty}$ form $g=u_{i}-\bar{\partial} v_{i}$ on $\mathbf{X}$ such that $\bar{\partial} g=f$.

Q. E. D.

Corollary 4.6 (Laufer [12], Kato [8]). Let $\mathbf{X}$ be a 1-convex manifold of dimension 2 with maximal compact analytic set $\mathbf{A}$, and let $\mathbf{L} \rightarrow \mathbf{X}$ be a line bundle. Assume that $\left.\mathbf{K}_{\mathbf{x}}^{*} \otimes \mathbf{L}\right|_{\mathbf{A}_{\mathbf{i}}}$ is of nonnegative degree for every irreducible component $\mathbf{A}_{\mathbf{i}}$ of $\mathbf{A}$. Then $\mathrm{H}^{1}(\mathbf{X}, \mathcal{O}(\mathbf{L})$ ) $=0$.

\section{§5. A Sufficient Condition for Rationality of Isolated Singularities}

Let $(\mathbf{X}, x)$ be a germ of an analytic space $\mathbf{X}$ for which $x$ is an isolated singular point. $(\mathbf{X}, x)$ is said to be rational if for any resolution of singularity $\pi: \widetilde{\mathbf{X}} \rightarrow \mathbf{X}, R^{q} \pi_{*} \mathcal{O}_{\widetilde{\mathbf{x}}}$ vanishes for $q \geqq 1$. Here $R^{q} \pi_{*} \mathcal{O}_{\tilde{\mathbf{x}}}$ denotes the higher direct image sheaves of $\mathcal{O}_{\tilde{\mathbf{x}}}$. Note that the property that $R^{q} \pi_{*} \mathcal{O}_{\tilde{\mathbf{x}}}=0$ for $q \geqq 1$ is independent of the choice of the resolution. (cf. Hironaka [6]). We can state a condition for the rationality of $(\mathbf{X}, x)$ in terms of the maximal compact analytic set of $\tilde{\mathbf{X}}$.

Theorem 5.1. Let the notation be as above and let $\mathrm{A}$ be the maximal compact analytic subset of $\widetilde{\mathbf{X}}$. Assume that $\mathbf{K}_{\tilde{\mathbf{x}} \mid \mathbf{A}}$ has a metric $h$ along the fibers for which $\left(\mathbf{K}_{\tilde{\mathbf{x}} \mid \mathbf{A}}, h\right)$ is Nakano-semipositive. Then $(\mathbf{X}, x)$ is rational.

Proof is immediate from Theorem 4.5.

As an application we obtain the following

Proposition 5.2. Let $\mathbf{X}$ be an analytic space of dimension 3 with an isolated singularity at $x$. Let $\pi: \widetilde{\mathbf{X}} \rightarrow \mathbf{X}$ be a resolution of singularity. Suppose that $\mathbf{A}=\pi^{-1}(x)$ is isomorphic to $\mathbf{P}^{1}$ and that the normal bundle of $\mathbf{A}$ splits into line bundles whose chern classes are either $(-1,-1),(-2,0)$, or $(-3,1)$. Then, $(\mathbf{X}, x)$ is a rational singularity. 
The following proposition was suggested by A. Fujiki.

Proposition 5.3. Let $\mathbf{X}$ be an analytic space of dimension 3 with a rational isolated singularity at $x$. Let $\pi: \widetilde{\mathbf{X}} \rightarrow \mathbf{X}$ be a resolution of the singularity. Suppose that $\mathbf{A}=\pi^{-1}(x)$ is isomorphic to $\mathbb{P}^{1}$ and that the degree of $\mathbf{K}_{\widetilde{\mathbf{x}} \mid \mathbf{A}}$ is zero. Then there exist a neighbourhood $U$ of $x$ and a nowhere-zero holomorphic 3-form defined on $U-\{x\}$.

\section{Proof is standard.}

Combining Proposition 5.2 with Proposition 5.3 we obtain the converse of the following

Theorem 5.4 (Theorem 4.1 in Laufer [13]). Let $\mathbf{X}$ be an analytic space of dimension $n \geqq 3$ with an isolated singularity at $x$. Suppose that there exists a nowhere zero holomorphic $n$-form $\omega$ on $\mathbf{X}-x$. Let $\pi: \widetilde{\mathbf{X}} \rightarrow \mathbf{X}$ be a resolution. Suppose that $\mathbf{A}=\pi^{-1}(x)$ is 1-dimensional and irreducible. Then $\mathbf{A}$ is isomorphic to $\mathbb{P}^{1}$ and $n=3$. Also, the normal bundle of $\mathbf{A}$ splits into line bundles whose chern classes are $(-1,-1),(-2,0)$, or $(-3,1)$.

\section{References}

[1] Andreotti, A. and Vesentini, E., Carleman estimates for the Lapalace-Beltrami equation on complex manifolds, Publ. Math. I. H.E.S. 25 (1965), 81-130.

[2] Fujiki, A. and Nakano, S., Supplement to "On the inverse of monoidal transformation", Publ. RIMS, Kyoto Univ. 7 (1971-72), 637-644.

[ 3 ] Fujita, T., On Kähler fiber spaces over curves, J. Math. Soc. Japan. 30 (1978), 779-794.

[4] Grauert, H., On Levi's problem and the imbedding of real-analytic manifolds, Ann. of Math. 68 (1958), 460-472.

[5] Grauert, H. and Riemenschneider, O., Kählersche Mannigfaltigkeiten mit hyper-q-konvexem Rand, Problems in Analysis, Princeton, N. J.: Princeton University Press 1970.

[6] Hironaka, H., Resolution of singularities of an algebraic variety over a field of characteristic zero I, II, Ann. of Math. 79 (1964), 109-326.

[ 7 ] Hironaka, H. and Rossi, H., On the equivalence of imbeddings of exceptional complex spaces, Ann. of Math. 156 (1964), 313-333.

[8] Kato, M., Riemann-Roch theorem for strongly pseudoconvex manifolds of dimension 2, Ann. of Mate. 222 (1976), 243-250.

[9] Kazama, H., Approximation theorem and application to Nakano's vanishing theorem for weakly 1-complete manifolds, Mem. Fac. Sci. Kyushu Univ. 27 (1973), 221-240.

[10] Kodaira, K., On a differential-geometric method in the theory of analytic stacks, Proceedings of the National Academy of Sciences, U.S. A. 39 (1953), 1268-1273.

[11] - On Kahler varieties of restricted type, Ann. of Math. 60 (1954), 28-48.

[12] Laufer, H., On rational singularities, Amer. J. Muth. 94 (1972), 597-608. 
[13] Laufer, H., On $\mathbf{C P}^{1}$ as an exceptional set, Recent developements in several complex variables, Ann. of Math. Stud. Princeton University Press, 100, 1981.

[14] Nakano, S., On the inverse of monoidal transformation, Publ. RIMS, Kyoto Univ. 6 (1970/71), 238-254.

[15] - Vanishing theorems for weakly 1-complete manifolds, Number theory, algebraic geometry and commutative algebra, Kinokuniya, Tokyo, 1973.

[16] Ohsawa, T., On complete Kähler domains with $\mathrm{C}^{1}$-boundary, Publ. RIMS, Kyoto Univ. 15 (1980), 929-940.

[17] - Isomorphism theorems for cohomology groups of weakly 1-complete manifolds, Publ. RIMS, Kyoto Univ. 18 (1982), 191-232.

[18] Vesentini, E., Lectures on Levi convexity of complex manifolds and cohomology vanishing theorems, Tata Inst. of Fundamental Research, Bombay, 1967.

Added in proof. Combining Proposition 5.3 with a result of M. Reid (Minimal models of canonical 3-folds, Proc. Sympos. Algebraic and Analytic Varieties (Tokyo, June 1981), Sympos. in Math, vol. 1, Kinokuniya, Tokyo and North-Holland, Amsterdam), Proposition 5.2 is strengthened so that we can conclude that $(\mathbf{X}, x)$ is a hypersurface singularity with defining equation $z_{0}^{2}=f\left(z_{1}, z_{2}, z_{3}\right)$. The author is grateful to Dr. M. Tomari for informing Reid's result to him. 